\title{
The future of dentistry post-COVID-19: perspectives from Urgent Dental Care centre staff in England
}

\author{
Robert Witton, ${ }^{\star 1}$ Anastasios Plessas, ${ }^{1}$ Hannah Wheat, ${ }^{2}$ Rebecca Baines, ${ }^{3}$ Maria Bernardes Delgado, ${ }^{1}$ Ian Mills ${ }^{1}$ and \\ Martha Paisi ${ }^{1}$
}

\section{Key points}

Reports the potential implications of COVID-19 on workforce sustainability and dental access from the perspective of UDC staff in England.
Research findings highlight a desire from the dental profession for progress in transforming dental services, particularly dental contract reform in England.
Key recommendations identified were: 'levelling up' NHS dental access; support for practices; a focus on recruitment and retention of the dental team; mental health wellness support; addressing future training needs; integrating oral health into wider healthcare; and replacement of the UDA system as part of dental contract reform, with a focus on prevention.

\section{Abstract \\ Introduction While routine dentistry was suspended during the COVID-19 pandemic, Urgent Dental Care centres (UDCs) were established to support patients with urgent dental needs.}

Aim The study aimed to provide insights and recommendations on future dental reform based on the perspectives of frontline staff delivering dental care in UDCs.

Method A qualitative research study using a phenomenological approach. A hybrid approach that incorporated both a deductive and an inductive approach to analysis was adopted.

Results The sample included 29 dentists and nine dental nurses providing care at UDCs in England during the pandemic. The following predominant themes were identified: patient care and access, practice viability, workforce sustainability, identity of dentistry and contract reform.

Conclusions The pandemic has highlighted the desire from the dental profession for urgent reform of the current NHS dental contract in England. This was directed towards a more resilient, responsive and equitable dental service that is better prepared for current and future challenges.

\section{Introduction}

The unprecedented nature of the SARSCoV-2 (COVID-19) pandemic has caused exceptional disruption to dental services. It has been particularly challenging for the dental profession and for patients accessing oral healthcare services, due to the risks of SARSCoV-2 in a dental setting. ${ }^{1}$ In order to support urgent care needs, Urgent Dental Care centres (UDCs) were established at the outset of the pandemic to provide urgent care during the lockdown periods. UDCs continue to operate

'University of Plymouth, Peninsula Dental School, Plymouth, UK; 2 University of Plymouth, Peninsula Medical School, Plymouth, UK; ${ }^{3}$ University of Plymouth, Centre for

Health Technology, Plymouth, UK.

${ }^{*}$ Correspondence to: Robert Witton

Email address: robert.witton@plymouth.ac.uk

Refereed Paper.

Accepted 22 July 2021

https://doi.org/10.1038/s41415-021-3405-1 as a network of service providers to support patient needs while recovery of routine dental services gathers pace.

The British Dental Association has estimated that 19 million fewer dental treatments had been delivered due to restrictions in routine dental care during the pandemic. ${ }^{2}$ The financial impacts of the pandemic on the business of dentistry have been a matter of ongoing concern for the dental profession and the pandemic has further exposed the limitations of the current dental contract in England, which ostensibly is not meeting the needs of patients or the dental profession. ${ }^{3}$ The Chief Dental Officer for England has identified the recovery from the pandemic as an ideal opportunity to transform dental services so they are oriented towards preventive care, address oral health needs more equitably, and capitalise on skill mix and innovation. ${ }^{4}$ In terms of transformation, there is a need to work together with all members of the dental team, and particularly those on the frontline working in UDCs, if we are to develop a fairer, more responsive dental system. However, to the best of our knowledge, no studies have so far explored the perspectives of UDC staff.

\section{Aim}

The aim of this paper is to identify recommendations for future dental reform in England based on insights provided by frontline staff delivering dental care in UDCs during the COVID-19 pandemic.

\section{Methods}

The methods used in the present study have been previously described. ${ }^{5}$ Briefly, this was a qualitative research study that adopted a phenomenological approach. ${ }^{6}$ A Senior Dental Officer working at a community dental service (CDS), and who provided care at a UDC, provided advice and input at all stages of the study including the research methods used. 
Participants were frontline staff at UDCs in England, including dentists and dental nurses. Participants were recruited through purposive sampling in the first instance and then snowballing and stratification. ${ }^{7,9}$

Online or telephone interviews of 45-60 minutes were conducted between June 2020 and August 2020 by a dental practitioner with extensive research experience. Interviews were digitally audio-recorded and transcribed verbatim. NVivo 12 software was used to support data coding and management.

A hybrid approach to thematic analysis was adopted. ${ }^{7,10,11}$ This incorporated both a deductive (top-down, theoretical) and an inductive (bottom-up, data-driven) process. Following a series of team meetings, themes were generated, defined and named.

\section{Ethical approval}

Ethical approval was obtained from the Faculty of Health and Human Sciences Research Ethics and Integrity Committee of the University of Plymouth (ref:19/20-1300). All participants consented to participate in the study and to have their data used as part of the research.

\section{Results}

Thirty-eight participants (29 dentists and nine dental nurses) took part in the interviews. Participant demographics have been previously described. ${ }^{5}$

The following themes were identified as important factors which needed to be addressed in order to support the future of NHS dentistry in England: backlog of patient care and dental access; practice sustainability; workforce sustainability and training needs; the identity of dentistry; and contract reform (Table 1).

\section{Backlog of patient care and dental access}

Participants reported that the COVID-19 pandemic had exacerbated current dental access problems: 'a lot of patients already find difficulty in registering with a dentist. I think that's going to be much worse now as well' (DCT1). A backlog of patients resulting from the pandemic and requiring treatment, described as 'a dental tsunami that's coming along' (DCT2), was perceived as an additional pressure facing NHS services, with detrimental impacts regarding future dental access. The need for 'managing patient expectations' (DN6) regarding waiting times to get a dental appointment was highlighted. Participants could also foresee that the 'already huge' (SD1) waiting lists for general anaesthesia, sedation, paediatric and special dental care services are 'going to go absolutely through the roof' (DN7), especially when considering such services are provided by the CDS, which still serve as UDCs today.

Participants were also concerned that patients may 'be reluctant to come back in because the perception of risk is still high for them' (CDS10). Delayed treatment-seeking behaviours raised concerns among participants regarding missing or late diagnosis of mouth cancer and unnecessary loss of teeth due to untreated disease: 'there'll be teeth there that could have been restored but that can no longer be restored' (CDS10).

While it was suggested that employing more staff could help alleviate the backlog of patients, limited numbers of facilities exacerbated by COVID-19 restrictions and existing difficulties in recruiting new staff could hinder this. A more realistic approach

Table 1 Theme and subtheme description table

Theme description
Backlog of patient care and dental access
The consequences of lockdown on patient access
and how this will impact on different areas of clinical
practice, such as: the impact on different patient
groups; missed or late diagnoses of dental disease
and oral cancer; the resource implications to address
the backlog alongside existing patient needs
Practice sustainability
The economic environment and concerns regarding
the easing of public health restrictions and how
this would impact business viability. Recognition
that COVID-19 has resulted in closure of dental
businesses

\section{Workforce sustainability}

The long-term implications on working conditions, particularly the mental health and wellbeing of dental professionals. Concerns regarding dental recruitment, retention of workforce and the consequences on dental education and graduate workforce due to disruption to training. Describes the education and training needs highlighted due to changes in clinical practice

\section{Identity of dentistry}

The role and purpose of dentistry and its integration in the wider healthcare system. The importance of oral health in general health and the focus on prevention

\section{Contract reform}

The ability of the dental contract to successfully address oral health needs in recovery and postpandemic, with suggestions for new models of care

\section{Subthemes}

- The impact of the lockdown on pre-existing dental access issues

- Lack of space to increase capacity

- Lack of funding to increase access and capacity

- The risks of recession and Brexit on business viability and job security

- Managing patient expectations regarding waiting times and level of service

\section{- Dentistry is more risky and more} stressful and the impacts are different on different dental team members

\section{Supporting quotes}

'We're trying to see our patients trying to get through the backlog. But to be honest, it's an impossible task...You know, we're seeing patients still really that are in terrible pain and we're not even scratching the surface yet' (DN9)

'But what this COVID pandemic has shown us is a huge cohort of patients that they cannot reach and access care' (GDP10)

I'm only doing eight people a day. You know, it's a fraction of what we were doing before we did 30 people a day. Yeah. How that will be sustainable financially in the future. I have no idea' (GDP3)

'We are gonna be entering a recession, patients won't be able to afford private. So those will also expect NHS treatments. But actually providing that is going to be a real challenge' (CDS10)

'For our FD trainee obviously there's going to be quite a, quite a big chunk of experience that he hasn't had. He's actually staying on with us as it [sic] part-time associate afterwards. So that really is an indication that although that's the case, I am still quite happy with him. But I think weaker FDs will struggle' (GDP12)

'I don't know how the universities are going to work around this [closure of student clinics due to COVID-19]' (DN6)

- A common risk factor approach

- Oral health in all policies

- Better public awareness campaigns

- Enhanced role for dental professionals in oral health advocacy

'Reconsidering other aspects like sugar tax that [sic] are talking about in terms of obesity. Let's get dentistry in on top of that so that we can influence oral health in that way' (CDS5)

'We need to be getting a more preventative approach. We need to be, you know, sort of factor in communication and education for patients. I think a lot of us have been saying this for years and years and years' (GDP13)

- Length of time contract reform is taking

- Contract currency
'This [contract] has to change, it can't carry on... we need to get rid of UDAs full stop' (GDP3)

'Part of it, of course, is contract reform, because the contract is laughable. And as we all know the contract was never going to work even before it was instituted. Here we are 15 years on, and we are still laughing about it very blackly' (CDS9) 
described both by community dentists and general dental practitioners (GDPs) was 'to prioritise people with highest need, either because of disease or because of cooperation and not being able to be treated normally or without any other techniques' (CDS6). Furthermore, some GDPs could see themselves extending the 'six-monthly review' to a 'yearly review' (GDP11) or 'doing a lot more in each session' (GDP13) to help them get through the backlog and deal with the fallow time restrictions.

\section{Practice sustainability}

Participants recognised that due to the COVID-19-related restrictions, 'dentistry is not going to get back to normal for a long time' (DN6) and 'the way we work will change beyond recognition' (GDP11). The dramatic decrease in capacity due to social distancing restrictions and fallow time requirements compounded by the increased 'amount and costs of PPE' (CDS2) were seen as particularly problematic for both NHS and private practices. Additional costs and restrictions in capacity were perceived as significant barriers in meeting existing service demands and keeping the 'business afloat' (CDS10), and would prevent additional access for new patients.

The current uncertain UK financial climate compounded by the potential 'Brexit' implications on economy (GDP7), along with the foreseeable bankruptcy of some dental laboratories and possible increase in unemployment, were seen to add to the aforementioned difficulties. In recognising the risk of a UK financial recession, some participants expressed the view that private practices may lose patients, placing further pressure on NHS services.

\section{Workforce sustainability}

An escalated focus on how to sustain and support both the current and future dental workforce was considered imperative by participants.

\section{Existing workforce}

Participants acknowledged that there might be long-term implications for workforce sustainability given the pressures faced by UDC staff members, furloughed staff members and people making career changes due to the difficulties encountered. Participants could foresee that 'a lot of professionals and dentists are going to leave' (DN6) and 'go for a career change' (GDP11), while some may decide to retire early. Similar concerns were also raised for newly qualified dentists and dental nurses, with a lack of available dental nurses who 'want to come into dentistry' (DN2) also frequently reported. Participants identified recruiting more dentists and dental nurses as an area of importance moving forwards.

Given perceived inadequacies in dental recruitment and retention before COVID19 , and increased risk of burnout due to COVID-19-related pressures, the importance of supporting the health and wellbeing of the existing workforce (including those returning from furlough) was repeatedly emphasised by participants: 'I just hope that all this concern about our health and wellbeing going forward is as well recognised as it is now' (CDS10).

\section{Future workforce}

Linked to the sustainability of the existing workforce were also concerns of the impact of COVID-19 on current undergraduate students and the future dental workforce; for example: 'for our trainees, it's an absolute disaster' (SD1); 'it may have compromised many people's training' (DCT2). For some participants, an inability to provide sufficient training and experience because of COVID19 was considered to be detrimental to patient care and long-term sustainability of dentistry more broadly.

A risk of deskilling was also noted in areas such as restorative dentistry and 'drilling skills' (CDS11). As a result, several participants discussed the importance of training and ensuring a skilled multidisciplinary team. The training needs identified are presented in the online supplementary information.

\section{Identity of dentistry}

For some participants, operating within a UDC was seen as a 'wake-up call' (CDS9) for the profession to reconsider the underpinning ethos and purpose of dentistry: 'It's not about making people pretty. It is about making sure that people are not septic and not in paralysing amounts of pain...Dentistry is mostly about that' (CDS9).

Given current access issues to dental care within many areas of England, participants were unanimous in the need for dentistry to focus on patient education. The importance of enhancing both knowledge and a sense of empowerment within the community setting (for example, 'delivering education to children, pregnant mothers, to the caregiver' GDP10) was seen as essential for increased prevention. However, to achieve this, participants called for dentistry, often described as 'the forgotten black sheep of the family' (GDP3), to be recognised as a healthcare service by both the government and existing systems, and to become 'integrated more into general healthcare' (GDP11). Participants highlighted that the government should take full advantage of the unique role that dentistry holds in tackling public health problems such as obesity and diabetes, by adopting a common risk factor approach: 'concerning our role in diabetes, our role in diet advice about sugars. Let's get dentistry in on top of that so that we can influence oral health in that way' (CDS6).

\section{Contract reform}

The most frequently suggested area for improvement suggested by participants was 'contract reform' (GDP7). This related to both short- and long-term ambition, recognising the immediate pressures faced by UDCs:

- 'I think so short term, the NHS should still be funding dentists, giving them the full contract, even though they're not making UDAs' (CDS2)

- 'The future dentistry needs a new NHS contract, that's what it needs, we can't have the same one we've got. It has to go, there has to be a new one' (GDP3).

Many participants acknowledged that a 'return to UDAs' (GDP6) was not feasible, 'viable' (CDS2), or appropriate given the significant reduction in capacity and increased costs. Units of Dental Activity (UDAs) were also often viewed as unsustainable before COVID-19 by both general dental practice and the CDS: 'I think a lot of people would be very glad to see the end of UDAs, I don't think they've done what they were supposed to do, I think it's driven people out of the profession, it's caused unnecessary stress' (GDP13). As a result, many participants viewed the pandemic as 'a real opportunity to change' (CDS10) and encourage innovative contract reform: 'I'm hoping it's actually going to become a force for positive change...change is long overdue' (CDS11). Several participants also called for a more participatory rather than a 'commanding approach' (GDP7) in reshaping the future of NHS dentistry.

Recognising a need for 'new ways' (GDP5) of measuring activity, some participants suggested a move towards a 'core service' (DN2). This was seen as a more viable solution for both patients and dentists, with a need to consider potential inequalities or difficulties 
in accessing such services for certain patient groups; for example, those with learning disabilities. Some participants also described a 'capitation-based'(GDP5) contract. However, it was considered to be unlikely that such a service would ever be implemented due to the focus on activity-based systems. Another suggestion was a 'salaried service' (GDP2) which may be seen as desirable, especially by 'associates' (GDP3), due to the 'security' (CDS10) and 'protection that you get from being a PAYE' (GDP3).

\section{Discussion}

Viral pandemics are not new and it is almost certain the dental profession will need to respond to the challenge of public health emergencies in the future - including further COVID-19 waves. The current COVID-19 pandemic has resulted in the most dramatic and significant disruption to dental services in a generation, and the implications for oral health and the dental profession are still emerging. ${ }^{2,3}$ In future, greater emphasis on robust emergency preparedness planning at a national level could ensure dental services are not compromised to the same extent as they were in this pandemic response. ${ }^{2,3,5}$ Greater regional support and guidance for dental organisations and practices in business continuity planning to create a more resilient and responsive dental system will also be required. This will need to include workforce planning to ensure that in future emergency scenarios, there is sufficient dental workforce available to be mobilised in a time-responsive and systematic way, alongside the necessary stocks of emergency personal protective equipment and stress-tested preparedness protocols and plans.

The backlog of patient care and the impact this could have on dental access moving forwards was highlighted throughout the study. There was universal recognition that the pandemic has exposed longstanding dental access issues and could further worsen the situation, a concern also shared by professional bodies. ${ }^{2,3}$ Of particular concern were patients normally cared for by CDS, recognising that dental risk is higher in patients with additional needs or in patients from socially excluded groups. Considering the perceived 'unfairness in the system' expressed by clinicians working in CDS, in future, NHS emergency preparedness should reduce reliance on these services where provision of dental care is restricted and adopt a more distributed model of urgent care where possible. This would enable more rapid recovery of services for those patients that are vulnerable and high-risk.

Participants in this study also reported concerns about access to referral services for children and the implications this might have for vulnerable children. Children's oral health is particularly at risk due to the cancellation of network services during the pandemic, such as elective tooth extraction under general anaesthesia, and the suspension of community health services such as health visiting, school nurse activities and community oral health improvement programmes. ${ }^{12}$ Greater investment in child oral health programmes post-pandemic, such as supervised toothbrushing schemes and better integration of oral health into wider children's services, will be required if the gains in children's oral health made over recent years are not to be lost.

The arduous nature of working through the pandemic was apparent in participant accounts and has been reported in detail in this study, ${ }^{5}$ and by others during the lockdown. ${ }^{13,14}$ Focusing on the physical and mental wellbeing of all staff members involved was considered imperative, given perceived inadequacies with such processes before COVID-19. ${ }^{5}$ Providing a working environment that retains talent and supply of workforce within the NHS dental system will be of paramount importance if dental services are to fully recover to prepandemic levels, in addition to addressing the backlog of patients. There is an ongoing need to understand the stressors and mental health implications across all dental team members resulting from the pandemic and the consequent effect on workforce. Occupational health and wellbeing of dental team members is an area requiring further research and policy prioritisation, not just in relation to the pandemic but also in response to the regulatory and performance framework within which dentistry operates. ${ }^{15}$

Dental contract reform was a key recommendation to improve the future, linking together and overlapping all of the themes generated. Although contract reform is underway, there was fatigue and frustration expressed by participants in this study concerning the length of time contract reform is taking. A contract that meets the needs of patients and the profession alike was seen as key for improved access, quality and health outcomes. In turn, participants felt this would positively influence workforce retention, practice stability and improve the integration of dental care within the wider healthcare system. The pandemic has clearly exposed the weaknesses and limitations in the current NHS dental contract in England. This has been reported elsewhere, and in particular has highlighted dissatisfaction among GDPs with the continued use of UDA as the only contract currency. ${ }^{16}$ There is also emerging evidence that the current contract may promote oral health inequalities between fee-paying and fee-exempt adult patients, with a decrease in fee-exempt adult patient attendance since the introduction of the contract in 2006. ${ }^{17}$ Although the new contract models proposed could deliver improvements in access, prevention and patient satisfaction, many challenges still exist in their costeffectiveness and ability to engage high-needs patients. ${ }^{18,19}$ Facilitating better integration and communication channels with other healthcare providers would also deliver benefits to patient care, increased efficiency and improvements in the working conditions of dental teams. ${ }^{5}$

A universal core service to address oral health inequalities may be a more appropriate way of levelling up the inequity in dental access and outcomes. The social contract between the public and the NHS for provision of dental services requires re-evaluation and a more honest and open dialogue with the public regarding the availability and scope of NHS dentistry. While we acknowledge that dental contract reform is currently underway, there is an urgent need to reinvigorate and move with pace to prevent further deterioration in access and protect the oral health of the most vulnerable.

\section{Conclusions}

The results of this study add the real-world view of dental staff during the pandemic, and capture the participants' views and experiences of delivering dental care in extremely challenging circumstances. These accounts, which have generated a number of important themes for consideration, are valuable in supporting dental reform and future resilience planning.

Not surprisingly, dental contract reform featured as one of the most frequent suggestions for improving working conditions for the dental team and for meeting the needs of patients, with a greater desire for prevention both at the chairside and outside in the 
community. This is not new, and the challenge remains as to how this can be achieved working together as a profession for better oral health within the constraints of the NHS system and particularly for those patients most at risk.

\section{Ethics declaration}

The authors declare no conflicts of interest.

\section{Acknowledgements}

We would like to thank our participants who engaged with our research and shared their views and experiences with us. The authors would like to thank all organisations, the journals and individuals who kindly cascaded the information about our research study. Funding: Peninsula Dental Social Enterprise (PDSE) partially funded this study.

\section{Author contributions}

Study conceptualisation: the study was conceptualised by MP, AP, RW and MBD. Interview guide: the interview guide was initially developed by $M P, A P$ and RW. The interview guide was then amended and finalised after receiving critical feedback by MBD, HW and IM. Data collection: the interviews were conducted and transcribed verbatim by AP. Data analysis: coding and identification of emerging themes - five interviews were independently coded by $A P, M B D$ and $R B$. Coding results were compared in a team meeting, overseen by an experienced qualitative researcher (HW), and emerging themes were identified and discussed. $R B$, who has extensive qualitative research experience, then coded half of the interviews. Sense checking was performed to review the analysis and to question the rationale for decisions (RB, $A P, H W, M P)$. Subsequently, all interviews were coded by $R B$ and codes and subthemes were discussed with HW, MP, AP and RW. Refining and defining of themes: finally, the emergent themes were generated, defined and named by AP, MP, HW and $R B$. Draft manuscript: the draft manuscript was jointly prepared by AP and MP. The manuscript was finalised after receiving critical feedback by $H W, R B$, $R W, M B D$ and $I M$.

\section{References}

1. Public Health England. COVID-19: Guidance for maintaining services within health and care settings - Infection prevention and control recommendations. 2021. Available at https://assets.publishing.service.gov. uk/government/uploads/system/uploads/attachment_ data/file/954690/Infection_Prevention_and_Control_ Guidance_January_2021.pdf(accessed April 2021).

2. British Dental Association. Dentists: Government holds the key to restore services to millions. 2020. Available at https://bda.org/news-centre/press-releases/dentistsgovernment-holds-the-key-to-restore-services-tomillions (accessed April 2021).

3. Westgarth D. How much longer does NHS dentistry have left? BDJ In Pract 2020; 33: 12-15.

4. Hurley $S$. Why re-invent the wheel if you've run out of road? Br Dent J 2020; 228: 755-756.

5. Plessas A, Paisi M, Baines R et al. Frontline experiences and perceptions of Urgent Dental Care centre staff in England during the COVID-19 pandemic: a qualitative study. Br Dent J 2021; DOI: 10.1038/s41415-021-3375-3.

6. Lin C S. Revealing the "Essence" of Things: Using Phenomenology in LIS Research. Qual Quant Methods Libr 2017; 2: 469-478.
7. Braun V, Clarke V. Successful qualitative research: A practical guide for beginners. London: SAGE, 2013.

8. Ritchie J, Lewis J, Elam G. Designing and selecting samples. In Ritchie J, Lewis J (eds) Qualitative Research Practice. pp 77-108. London: SAGE, 2003.

9. Barbour R. Introducing qualitative research: a student's guide. 2nd ed. London: SAGE, 2014

10. Braun V, Clarke V. Using thematic analysis in psychology. Qual Res Psychol 2006; 3: 101-177.

11. Swain J. A Hybrid Approach to Thematic Analysis in Qualitative Research: Using a Practical Example. London: SAGE, 2018.

12. Okike I, Reid A, Woonsam K Dickenson A COVID19 and the impact on child dental services in the UK. BMJ Paediatr Open 2021; DOI: 10.1136/ bmjpo-2020-000853.

13. Collin V, O'Selmo E, Whitehead P. Psychological distress and the perceived impact of the COVID-19 pandemic on UK dentists during a national lockdown. Br Dent J 2021; DOI: 10.1038/s41415-020-2592-5.

14. Mahendran K, Patel S, Sproat C. Psychosocial effects of the COVID-19 pandemic on staff in a dental teaching hospital. Br Dent J 2020; 229: 127-132.

15. Toon M, Collin V, Whitehead P, Reynolds L. An analysis of stress and burnout in UK general dental practitioners: subdimensions and causes. Br Dent J 2019; DOI: 10.1038/sj.bdj.2019.46.

16. Holmes R D, Steele J G, Donaldson C, Exley C. Learning from contract change in primary care dentistry: $A$ qualitative study of stakeholders in the north of England. Health Policy 2015; 119: 1218-1225.

17. Shah S, Wordley V. An overview of adult dental fee exemptions in NHS primary dental care in England. $\mathrm{Br}$ Dent J 2021; DOI: 10.1038/s41415-021-2790-9.

18. Hulme C, Robinson P G, Saloniki E C et al. Shaping dental contract reform: a clinical and cost-effective analysis of incentive-driven commissioning for improved oral health in primary dental care. BMJ Open 2016; DOI: 10.1136/bmjopen-2016-013549.

19. Robinson P G, Douglas G V A, Gibson B J et al. Remuneration of primary dental care in England: a qualitative framework analysis of perspectives of a new service delivery model incorporating incentives for improved access, quality and health outcomes. BMJ Open 2019; DOI: 10.1136/bmjopen-2019-031886. 